ORIGINAL ARTICLE

\title{
Utilization Status of Electronic Information Sources (EIS) for HIV/AIDS Care and Treatment in Specialized Teaching Hospitals of Ethiopia, 2016
}

\author{
Senait Samuel Bramo ${ }^{1}$, Tesfamichael Alaro Agago $^{2 *}$
}

\author{
OPEN ACCESS \\ Citation: Senait Samuel Bramo, \\ Tesfamichael Alaro Agago. Utilization \\ Status of Electronic Information Sources \\ (EIS) for HIV/AIDS Care and Treatment \\ in Specialized Teaching Hospitals of \\ Ethiopia, 2016. J Health Sci \\ 2017;27(5):507. \\ doi:http://dx.doi.org/10.4314/ejhs.v27i5.9 \\ Received: March 12, 2017 \\ Accepted: March 16, 2017 \\ Published: September 1, 2017 \\ Copyright: () 2017 Senait Samuel \\ Bramo., et al. This is an open access \\ article distributed under the terms of the \\ Creative Commons Attribution License, \\ which permits unrestricted use, \\ distribution, and reproduction in any \\ medium, provided the original author and \\ source are credited. \\ Funding: Jimma University, College of \\ Natural Science \\ Competing Interests: The authors \\ declare that this manuscript was approved \\ by all authors in its form and that no \\ competing interest exists. \\ Affiliation and Correspondence: \\ ${ }^{1}$ Jimma University College of Natural \\ Science Department of Information \\ Science \\ ${ }^{2}$ Jimma University Institute of Health \\ Sciences Department of Health \\ Economics, Management and Policy \\ *Email: tesfamichaelalaro@yahoo.com
}

\begin{abstract}
BACKGROUND: According to the World Health Organization, the use of Electronic Information Sources (EIS) in healthcare is not merely about application of technology, but it is also a foundation to provide higher quality clinical care. This study was aimed to assess Utilization Status of EIS for HIV/AIDS Care and Treatment in specialized teaching hospitals of Ethiopia, 2016.

MATERIALS AND METHODS: A facility based cross-sectional study design was used. The study populations were 352 healthcare professionals selected by using simple random sampling technique from three randomly selected specialized teaching hospitals of Ethiopia. Quantitative and qualitative data were collected and analyzed by fitting multivariate logistic regression model and thematically by bringing similar themes together respectively.

RESULTS: This study revealed that only $33.2 \%$ of the health professionals used EIS as supporting tool in their clinical practice including HIVIAIDS care and treatment. The main reasons for not using EIS were having no training 285(89.9\%), followed by preferring print resources $20(6.3 \%)$. Furthermore, there was statistically significant association between use of EIS and perceived electronic information retrieval skills $A O R=3.271, C I$ (1.942, 4.051), perceived quality of electronic information content retrieved $A O R=2.069, C I(1.051,3.925)$ and limited access to computer and internet connection $A O R=5.072, C I(1.834,5.931)$. CONCLUSIONS: In this study, only one-third of health professionals used EIS as supporting tool in their clinical practice. Hence, hospital boards should devise strategies to improve utilization of EIS.

KEYWORDS: Electronic Information Source, Ethiopia, HIV/AIDS, Specialized Teaching Hospital
\end{abstract}

\section{INTRODUCTION}

The shift from printed forms of information resources to Electronic Information Sources (EIS) lead to better quality and efficient and effective delivery of healthcare by health professionals. According to the World Health Organization (WHO), the use of EIS in healthcare is not merely about technology. Furthermore, it's fundamental in 
making better treatment decisions, providing higher quality and safer care, supporting the development of effective, efficient and equitable health systems. (1). The use of EIS enables health professionals to effectively and efficiently access digital information to assist in investigating issues, solving problems, making decisions and creative solutions to support learning and develop new understanding in areas related with HIV/AIDS care and treatment (2).

The use of EIS brought speedy access to many different resources, professional development opportunities and time saving (3). Moreover, harnessing Information Technology (IT) empowers learning and clinical services, enhances cognitive skills for critical inquiry, and strengthens professional identity and values $(4,5)$.

EIS is accessed from a variety of sources, including websites run by organizations, homepages run by individuals, and online support groups where people actively exchange health information and blogs (6).Several countries, including Ethiopia and Mali, have made notable advances in utilizing Information Communication Technology (ICT) to increase healthcare access and quality of service for their populations (7). Hence, healthcare professionals play a vital role in improving the health outcomes, quality of care, and the health care experience of patients by utilizing the technology.

Application of ICT as EIS tool has brought breakthrough towards addressing the HIV and AIDS pandemic through spreading education/awareness, patient management and treatment management. Moreover, an ICT application specifically for HIV/AIDS has provided opportunities for social and behavioral change and for raising awareness on health issues (8).

HIV/AIDS treatment and management strategies require on-going monitoring and evaluation, and e-health systems have been recommended as a supporting tool $(9,10)$. One of the challenges for access to treatment through anti-retroviral medication is the difficulty of receiving accurate and up-to-date information at the planning level (11). HIV/AIDS care and treatment to poor communities can be significantly improved by integrating local primary healthcare information with centralized databases to allow national monitoring (1).

As more information resources are being made available electronically (12). Issues of training are important if healthcare professionals do not possess sufficient skills to access EIS. Most importantly, lack of computer and information retrieval skills lead to their underutilization (13). There are a number of EIS that healthcare professionals can utilize to offer services in the best interests of patients and the community. However, some of the available EIS have not been utilized at all (14), and access to information sources may be delayed and not accessible when needed (12).

HIV/AIDS care and treatment is one of the health services delivered in specialized teaching hospitals of Ethiopia. So far, little evidence has been documented on the use of even the available EIS for mitigating HIV/AIDS in Ethiopia as evidenced by the Ministry of Health report and the Federal HIV/AIDS Prevention and Control (15). Hence, this research provided evidence on the level of EIS utilization on HIV/AIDS care and treatment and identified factors affecting use of EIS. Hence, it will help to close the evidence gap on the EIS use for HIV/AIDS care and treatment in specialized teaching hospitals in Ethiopia.

\section{MATERIALS AND METHODS}

The study was conducted in three specialized teaching referral Hospitals of Ethiopia, namely, Jimma University Specialized Teaching Hospital in Jimma Zone, Oromia Regional State, Hawassa Referral Teaching Hospital in Hawassa, Southern Nations, Nationality and Peoples (SNNPR) Regional State and Black Lion Specialized Hospital in Addis Ababa City Administration from Feb 14, 2016 to May 2, 2016.

A facility based cross-sectional study design with both quantitative and qualitative data collection method was employed. The sample size was calculated using single population proportion formula where, proportion of health professionals who had access to EIS was $50 \% \quad(\mathrm{p}=0.5)$. Accordingly, the final sample size for this study

DOI: http://dx.doi.org/10.4314/ejhs.v27i5.9 
was calculated to be 352 health professionals. The total sample was proportionally allocated to respective hospitals based on the size of health professionals, and from each hospitals, health professionals were selected by using simple random sampling technique.

Quantitative data were collected using a structured self-administered questionnaire by twelve data collectors and three supervisors. The qualitative data were obtained from the key informant interviews with Anti Retroviral Therapy (ART) clinic focal person, clinical director of the hospital) by using interview guide. Quantitative data were entered using EPIDATA version 3.0 software. Data were transported to SPSS version 17.0 statistical package for further analysis. Ethical clearance was obtained from Institutional Review Board of the College of Natural Science, Jimma University. Proportions and numbers were used for description of the results. Bivariate and multivariate logistic regression models were fitted to identify factors affecting use of EIS and 95\% Confidence interval (CI) with Adjusted Odds Ratio (AOR) were used to judge statistical significance associations between dependent and independent variables. Qualitative data were analyzed thematically by bringing similar themes together under each section (topics) of the interview guide to answer the research question.

\section{RESULT}

Socio-demographic characteristics of the study participants: Out of the total sampled populations, 317 were participated in the study with response rate of $90 \%$. Of the total health professionals participated in the study, 230(72.5 $\%$ ) were males. The mean age of the respondents was 23.3 years ranging from minimum 21 to maximum 54 with standard deviation of \pm 6.41 . Moreover, $182(57.4 \%)$ of the respondents were currently married and the mean family members were 2.927 with minimum 1 to maximum 6 . The majority of the respondents, $121(38.2 \%)$, were nurses followed by pharmacist, 102(32.2 \%). The mean length of work experience of the respondents was 4.400 years with minimum 1 to maximum 30 years (Table1).

Table 1: Socio-demographic characteristics of respondents in selected Specialized Teaching Hospitals of Ethiopia, 2016

\begin{tabular}{llll}
\hline Characteristics & & Number & Percent (\%) \\
\hline sex & Male & 230 & 72.5 \\
Religion & Female & 87 & 27.5 \\
& Orthodox & 111 & 35.0 \\
\multirow{5}{*}{ Marital status } & Protestant & 173 & 54.6 \\
& Muslim & 33 & 10.4 \\
\multirow{3}{*}{ Professional back-ground } & 125 & 39.4 \\
& Unmarried & 182 & 57.4 \\
& Currently married & 10 & 3.2 \\
& Widow/ widower & 6 & 1.9 \\
& Medical doctor with speciality & 19 & 6.0 \\
& Medical doctor & 121 & 38.2 \\
& Nurse & 102 & 32.2 \\
& Pharmacist & 43 & 13.6 \\
& Laboratory & 8 & 2.5 \\
& Radiology & 18 & 5.7
\end{tabular}

Electronic information sources use: Almost all, 309(97.5\%), of the health professionals were aware of the presence of HIV/AIDS resource centers in their hospitals. However, only $59(19.1 \%)$ of the health professionals reported that they visited the resource center regularly. For

DOI: http://dx.doi.org/10.4314/ejhs.v27i5.9 
example, medical director who was interviewed said, "Majority of healthcare professionals including me did not visit the resource center regularly because of poor internet connection and quality of electronic information contents retrieved is poor. On top of that the work is demanding, hence ...we do not of enough time to visit resources center".

More than three-fourth, 268(84.5\%), of the healthcare professionals ever used EIS. Accordingly, $99(36.9 \%)$ of the healthcare professionals used EIS for entertainment. Contrary to this, only $89(33.2 \%)$ used EIS to support their clinical practice. In line with this almost, threefourth of the respondents, $73.5 \%$ ), perceived that the quality of electronic information content retrieved was poor. However, an HIV/AIDS focal person said, "While I am using EIS; mainly I give concern to clinical practices including diagnosis and treatment of dermatological and oral manifestation of HIV/AIDS". Of the health professionals who ever used EIS, more than threefourth, $78.4 \%$, were not satisfied on the current use of EIS.

Regarding the types of EIS used for supporting clinical practice including HIV/AIDS care and treatment, this study revealed that $153(57.1 \%)$ preferred search engines like Google, yahoo, and wiki followed by audiovisual collection, 47(17.5\%) (Figure1). This finding was re-enforced by in-depth interview of a medical director who said, "Of the different EIS available I use search engine commonly Google even via private mobile phones due to the fact that it was user friendly". However, of the total respondents, 97(69.4\%) perceived their electronic information retrieval skills as poor.

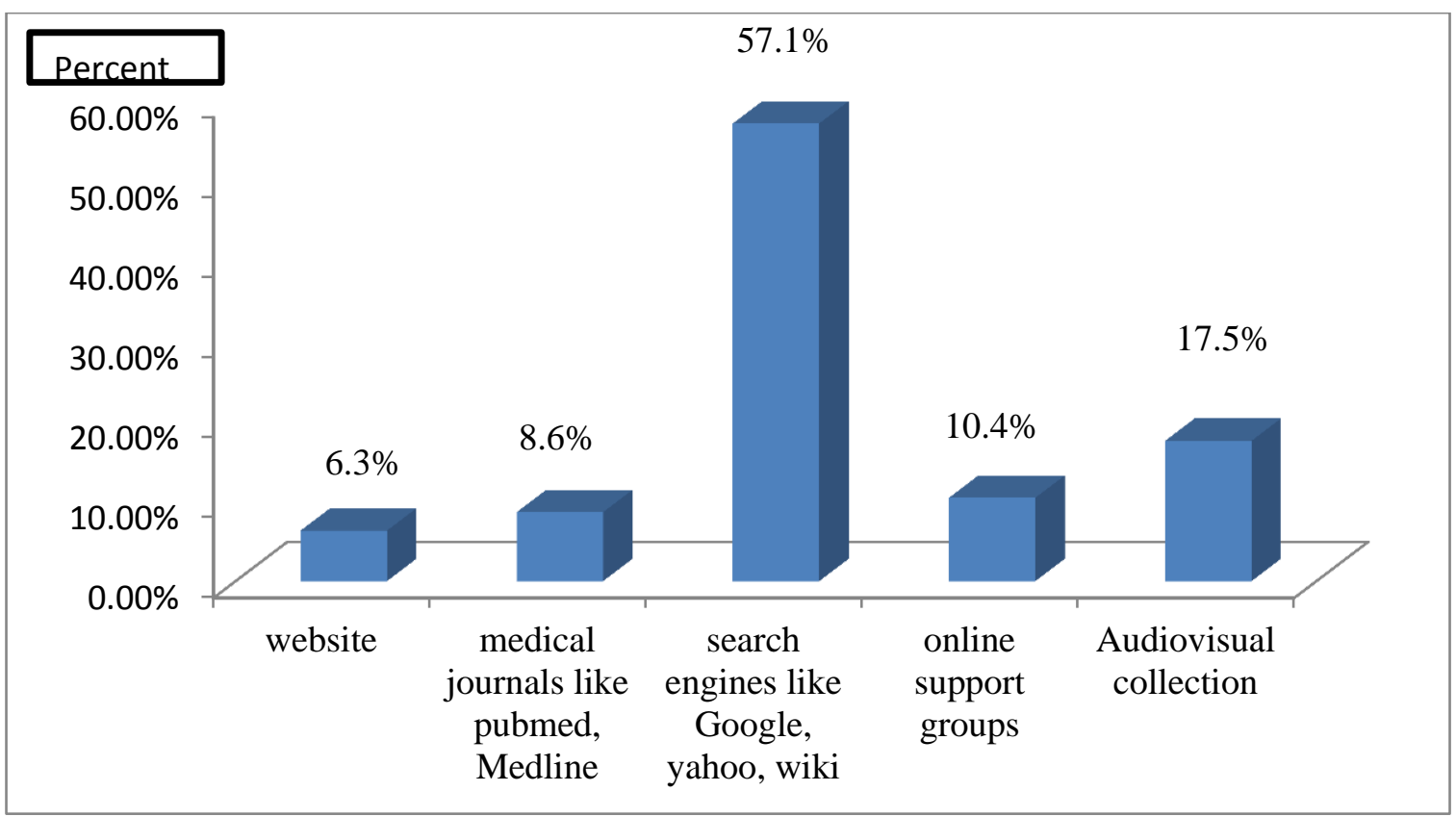

Figure 1: Type of electronic information resources preferred by health professionals, 2016

Of the total healthcare professionals who participated in this study,285(89.9\%) reported having no training as a reason for not using EIS for improving HIV/AIDS care and treatment followed by preferring print resources, 20(6.3\%) (Figure 2). Accordingly, a medical director interviewee said, "I never thought of using EIS because there is no promotion and training on how to access and use from the HIV/AIDS resource center and surprisingly most of health professional including me relay on our past memory and sometimes use print resources like guidelines and manuals as reference". Furthermore, another medical doctor working on ART said, “... there is no culture of visiting HIV/AIDS resource centers to use even the

DOI: http://dx.doi.org/10.4314/ejhs.v27i5.9 
available electronic information resources. Despite the fact, more information resources are being made available electronically, there is no training in our hospital on how to access and use EIS'.

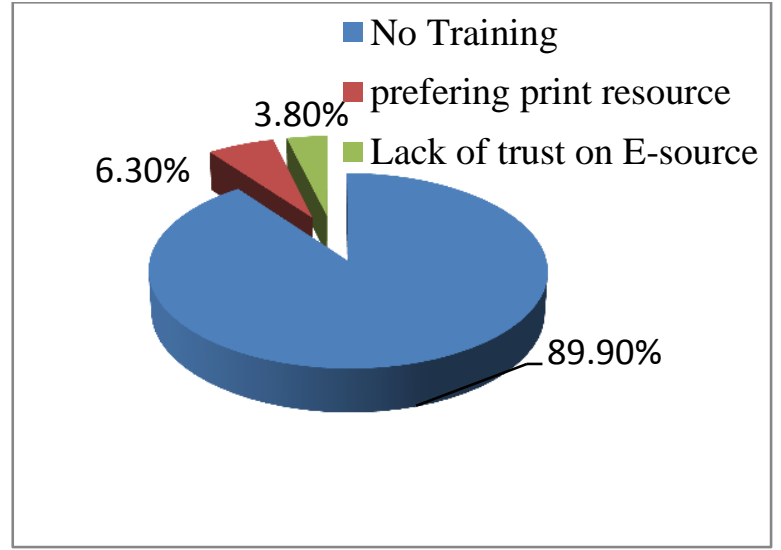

Figure 2: Reasons for not using Einformation sources in specialized teaching hospitals of Ethiopia, 2016

Factors Affecting Utilization EIS: To identify factors affecting use of EIS as supporting tool for clinical practice, variables were entered in stepwise into logistic regression model.
Accordingly, perceived electronic information retrieval skills, perceived quality of electronic information content retrieved, and limited access to computer and internet connection were found to be statistically significant factors associated with use of EIS as supporting tool for clinical practice at $95 \%$ CI (Table 2).

It was found that health professionals who perceived their electronic information retrieval skills as good were 3 times more likely to use EIS as supporting tool for clinical practice as compared to those who perceived poor, AOR = 3.271 , CI (1.942, 4.051). Health professionals who perceived the quality of the retrieved electronic information content as good were 2 times more likely to use EIS as compared to those who perceived it as poor, $\mathrm{AOR}=2.069$, CI (1.051, $3.925)$. In this study, the majority, 223(70.3\%), of the respondents had limited access to computer and internet connection. Interestingly, those who had access to computer and internet connection were 5 times more likely to use EIS as compared to those who had limited access to computer and internet connection, $\mathrm{AOR}=5.072, \mathrm{CI}(1.834$, 5.931) (Table 2).

Table 2: Factors affecting health professionals' use of EIS for HIV/AIDS care and treatment in selected specialized teaching hospitals of Ethiopia, 2016

\begin{tabular}{|c|c|c|c|c|}
\hline \multirow[t]{2}{*}{ Variable } & \multicolumn{2}{|l|}{ Use of EIS } & \multirow[t]{2}{*}{ COR $(95 \% \mathrm{CI})$} & \multirow[t]{2}{*}{$\operatorname{AOR}(95 \% \mathrm{CI})$} \\
\hline & $\begin{array}{l}\text { Yes } \\
\text { No }(\%)\end{array}$ & $\begin{array}{l}\text { No } \\
\text { No }(\%)\end{array}$ & & \\
\hline \multicolumn{5}{|c|}{$\begin{array}{l}\text { Perceived electronic } \\
\text { information retrieval skills }\end{array}$} \\
\hline Good & $89(91.8)$ & $8(8.2)$ & $1.351(1.486,2.372)$ & $3.271(1.942,4.051)^{¥}$ \\
\hline Poor * & $179(81.4)$ & $41(18.6)$ & 1 & 1 \\
\hline \multicolumn{5}{|c|}{$\begin{array}{l}\text { Perceived quality of electronic } \\
\text { information content retrieved }\end{array}$} \\
\hline Poor quality* & $216(92.7)$ & $17(7.3)$ & 1 & 1 \\
\hline Good quality & $52(61.9)$ & $32(38.1)$ & $1.852(1.032,1.984)$ & $2.069(1.051,3.925)^{¥}$ \\
\hline \multicolumn{5}{|c|}{$\begin{array}{l}\text { Access to computer and } \\
\text { internet connection }\end{array}$} \\
\hline Yes & $86(91.5)$ & $8(8.5)$ & $2.967(1.214,3.863)$ & $\begin{array}{l}5.072 \\
(1.834,5.931)^{¥}\end{array}$ \\
\hline No $*$ & $182(81.6)$ & $41(18.4)$ & 1 & 1 \\
\hline
\end{tabular}

DOI: http://dx.doi.org/10.4314/ejhs.v27i5.9 


\section{DISCUSSION}

This study revealed that $97.5 \%$ of the healthcare professionals working in the specialized teaching hospitals of Ethiopia were aware of the presence of HIV/AIDS resource centers at their hospitals. However, only one-fifth of the health professionals reported that they visited the resource center regularly to access EIS. This finding is comparable with the study done by Ibrahim in 2004, which reported that the frequency of use of EIS was low, 21.0\%, because of more time needed to focus on patients, lack of awareness about EIS provided by resource centers or libraries of the hospitals and ineffective communication channels (16).

Application of ICT as EIS tool has brought breakthrough in addressing the HIV and AIDS pandemic through spreading education/awareness, patient management and treatment management (8). In this study, about one-third of the health professionals used EIS as supporting tool in their clinical practice related with HIV/AIDS care and treatment. This finding is higher than the finding of a study done in Nigeria which reported that there was low usage of EIS among healthcare professionals in hospitals (1). The possible explanation for the observed discrepancy might be the booming of ICT in every sector including health and technical change and intensification of globalizationin (16). Even though there are recommendations that HIV/AIDS care and treatment to poor communities can be significantly improved by integrating local primary healthcare information with centralized databases to allow national monitoring (1), there was low (one-third of the health professionals) level of utilization of EIS.

This study found that the majority, $36.9 \%$, of the health professionals used EIS for entertainment. This is different from the finding of a study done in Malaysia by Nielson (2011) in which from 19 major search engines in the internet, health information ranked first most searched item (21). In contrast, this study is similar with a systematic review of 38 studies from 1994 to 2004 on why doctors use the internet which revealed that they focused on the use of email, retrieving information from online journals, attending courses and conferences, receiving professional updates and performing professional and administrative functions (17).

In this study, more than three-fourth of the healthcare professionals were not satisfied with the current utilization EIS. The finding is not in line with the study conducted by Masters $\mathrm{K}$, in Nigeria, which reported that $90 \%$ of respondents indicated that EIS had increased efficiency of their job. The possible explanation for the observed difference might be that, in the current study, the majority of the health professionals used EIS for entertainment not related to healthcare.

Issues of trainings are important if health professionals do not possess sufficient skills to access electronic resources. Moreover, lack of computer and information retrieval skills lead to underutilization EIS (19). Concurrently, the current study revealed that $88.9 \%$ of health professionals did not have any formal training on EIS utilization, and this negatively impacted on the usage of EIS for enhancing HIV/AIDS care and treatment. This finding is in line with the view of an in-depth interviewee who said, "... more information resources are being made available electronically but, there is no training in our hospital on how to access and use EIS". Furthermore, shortage of resources, like computers, and poor connection were reported as the main reasons hindering the use of EIS regularly for improving clinical practice including HIV/AIDS care and treatment. This is comparable with the finding of a study conducted by Gathoni in 2011 which reported that the common reasons highlighted for not using e-resources were poor connectivity and lack of computers (20).

In conclusion, only one-third of the health professionals used EIS as supporting tool in their clinical practice including HIV/AIDS care and treatment. Despite this fact, issues of trainings are important if health professionals do not possess sufficient skills to access electronic resources; in this study majority of the health professionals reported having no training as a reason for not using EIS. Hence, hospital boards should devise strategies to improve utilization by spotlighting the cultivation of culture of EIS utilization for

DOI: http://dx.doi.org/10.4314/ejhs.v27i5.9 
clinical service. Furthermore, due emphasis should be given for improvement of electronic information retrieval skills of health professionals and how to access and utilize computer and internet connection.

\section{ACKNOWLEDGEMENTS}

The authors would like to thank all of the participants of the survey for sharing their experience. Our gratitude extends to Jimma University, College of Natural Sciences, for funding the research as part of mega project.

\section{REFERENCES}

1. Dzenowagis, J. Bridging The Digital Divide: Linking Health and ICT Policy: Stud Health Technol Inform 2009. Accessed at 20/7/ 2015

2. Aramide. J and Bello. G. Use of Electronic Information Sources (EIS) by Faculty Members in Nigerian Universities. Library Philosophy and Practice (e journal) 2010. Accessed at 10/7/ 2015

3. Porumbeanu, O. L. The impact of electronic resources and new technology in academic medical libraries in Romania, Health Information and Libraries Journal 2009; 26: 152-155. Accessed at 21/7/ 2015

4. Naylor CD. Leadership in academic medicine: reflection from administrative exile. Clin Med 2006; 6: 488-93.

5. Saintz ML, Meleis AI. Integrating Cultural competence in to nursing education and practice: $21^{\text {st }}$ century action steps. $J$ TranscultNurs 2007; 18: 86s-90s.

6. Sillence E, Briggs P, Harris P, Fishwick L. (2007). Going Online for Health Advice: Changes in Usage and Trust Practices over the Last Five Years. Interacting with Computers; 19 (3):397-406.

7. World Health Organization. Information Communication Technology for Health in Africa. WHO 2013. Accessed at 16/7/ 2015

8. Shield's, Chetley.A and Davies. J. Improving Health, Connecting People: the role of ICT in the Health Sector in Developing Countries Summary of Online Discussions. Info Dev
Working Paper Series ICT and Health 2005. Accessed at 10/7/ 2015

9. Martı'nez A, Villarroel V, Seoane J, del Pozo F. Analysis of Information and Communication Needs in Rural Primary Healthcare in Developing Countries. IEEE Trans InfTechnol Biomed 2005; 9:66-72. Accessed at 20/7/ 2015

10. Norwegian Centre for Telemedicine. Workshop Summary. E-health Management for People Living with HIV/AIDS. Retrieved from http://www.telemed.no/ehealthmanagement-for-people-living-with-hivaids 2007. Accessed at 21/7/2015

11. Dorrington RE, Johnson LF, Bradshaw D, Daniel T. The Demographic Impact of HIV/AIDS in South Africa. National and International Indicators for 2006.Cape Town Centre for Actuarial Society of South Africa. Retrieved from http://www.commerce.uct.ac.za/Research_Uni ts/CARE/RESEARCH/ReportsWorkingPapers 2006. Accessed at13/7/2015

12. Brennan. P, Hersey.K and Harper.G. Strategic and practical Considerations for Signing Electronic Information Delivery Agreements 2002. Accessed at 15/7/2015

13. Mawindo, D. M. Evaluation of students' use of print and electronic resources at the University Of Malawi College Of Medicine. M.I.S. thesis. Pietermaritzburg: University of KwaZulu-Natal 2005. Accessed at 20/7/ 2015

14. Gakibayo,A., Ikoa-Odongo,J.R. \&OkelloObura,C. Electronic information resource utilization by student in mbarara University library. Library Philosophy and Practice (ejournal) 2013. Retrieved from http://digitalcommons.unl.edu/libphilprac/869

15. HIV/AIDS in Ethiopia an Epidemiological Synthesis. Ethiopia HIV/AIDS Prevention and Control Office (HAPCO) 2008.

16. Ibrahim, A. E. "Use and User Perception of Electronic Resources in the United Arab Emirates University (UAEU)," Libri 2004; 54:

18-29;

DOI: https://doi.org/10.1515/LIBR.2004.18 Accessed at 16/7/ 2015 
17. Thanuskodi. S. Electronic Information Sources and Services in Anna University Libraries in Tamil Nadu; International Journal of Library Science; Scientific \&Academic Publishing 2012; 1(3): 43-49; doi: 10.5923/j.library.20120103.01. Accessed at 22/7/ 2015.

18. Masters, K. For What Purpose and Reasons Do Doctors Use the Internet: a Systematic Review: International Journal of Medical Informatics 2008; 77: 4-16 Accessed at 23/7/ 2015

19. Olatokun. WM and Adeboyejo. OC. Information and Communication Technology Use by Reproductive Health Workers in Nigeria: State of the Art, Issues, and hallenges; An Interdisciplinary Journal on Humans in ICT Environments 2009.ISSN: 1795-6889; 5(2), 181-207v; Retrieved from: www.humantechnology.jyu.fi.

20. Gathoni. N, Gikandi. J, Ratanya. F, Njoroge.EM, Wasike.E, Kiilu. D and Kabugu. A. Monitoring and Evaluation of Electronic Resource in Academic and Research Institution in Kenya 2011. Accessed at23/7/ 2015

21. Nielson, Schardt. Connie. Health information literacy meets evidence-based practice. Journal of the Medical Library Association 2011; 99 (1): 1-2; doi: 10.3163/15365050.99.1.001 